\title{
Evaluation of Social Support, Quality of Life, and Body Image in Women with Breast Cancer
}

\author{
Roberta Spatuzzi $^{a}$ Anna Vespa ${ }^{b}$ Primo Lorenzi ${ }^{c}$ Guido Miccinesi ${ }^{d}$ Marcello Ricciutia \\ Wanda Cifarelli $^{\mathrm{e}}$ Marina Susi ${ }^{\mathrm{e}}$ Tommaso Fabrizio ${ }^{f}$ Maria G. Ferrarig Marica Ottaviani ${ }^{\mathrm{b}}$ \\ Maria V. Giulietti ${ }^{\mathrm{b}}$ Fabiana Merico $^{\mathrm{h}}$ Michele Aieta $^{\mathrm{i}}$ \\ a Palliative Care Unit, San Carlo Hospital, Potenza, Italy; \\ ${ }^{b}$ Department of Neurology, INRCA-IRCCS National Institute of Health and Science on Aging, Ancona, Italy; \\ ${ }^{\mathrm{C} C l i n i c a l}$ Psychology and Psychotherapy, University of Florence, Careggi Hospital, Florence, Italy; \\ ${ }^{d}$ Clinical and Descriptive Epidemiology Unit ISPO-Cancer Research and Prevention Institute, Florence, Italy; \\ e Department of Medical Oncology, Madonna delle Grazie Hospital, Matera, Italy; \\ ${ }^{f}$ Department of Plastic and Reconstructive Surgery, Oncology Referral Center of Basilicata, IRCCS, Rionero in Vulture, Potenza, Italy; \\ g Department of Neurological and Psychiatric Sciences, University of Florence, Italy;

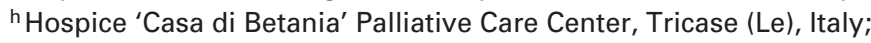 \\ iDepartment of Medical Oncology, Oncology Referral Center of Basilicata, IRCCS, Rionero in Vulture, Potenza, Italy
}

\section{Keywords}

Breast cancer - Quality of life · Body image .

Social support

\section{Summary}

Background: This study was aimed at comparing the quality of life, body image, and perceived social support in women with breast cancer surgery. Patients and Methods: Patients receiving breast-conserving surgery (BCS) ( $n=72$ ), mastectomy alone ( $n=44)$, and mastectomy with breast reconstruction ( $n=41$ ) were evaluated using the European Organization for Research and Treatment of Cancer Quality of Life Questionnaire (EORTC QLQ-C30), the EORTC Breast Cancer Module (QLOBR23), the Body Image Scale (BIS) and the Multidimensional Scale of Perceived Social Support (MSPSS). Results: The results indicated that the BCS group had a better body image compared with the other 2 groups and better role functioning compared with the mastectomyalone group. In the reconstruction group, body image correlated with perceived social support, especially from family and significant others. Conclusion: These results suggest that a positive perception of a supportive social network can help women with breast reconstruction to better cope with the psychological effects of surgery on their body image.

(c) 2016 S. Karger GmbH, Freiburg

\section{Introduction}

Breast cancer may be surgically treated in different ways: conservative surgery followed by irradiation [1], mastectomy alone, or mastectomy with immediate or delayed breast reconstruction. Because survival is the same in the 3 surgical procedures, the choice among them is often focused on deciding which one will optimize the quality of life (QoL) [2,3] and the body image (BI). Many studies have shown that the most frequently accounted factors for the difficulty to choose reconstruction were the lack of family support, the inability to have a specific type of reconstruction, and the perception that friends and acquaintances might have considered the surgery as cosmetic [4]. On the other hand, the most frequently reported reasons for having reconstruction included: to get rid of the external breast prosthesis, to be able to wear many different types of clothing, to regain femininity and to feel whole again $[5,6]$.

Multiple studies have demonstrated that women receiving breast-conserving surgery (BCS) generally report fewer difficulties with their BI but worse physical functioning than those treated by mastectomy alone, suggesting that side effects of adjuvant therapy and other short-term effects may also be important $[6,7]$. In fact, radiotherapy is known to have late effects on normal tissues, which could contribute adversely to the BI and related QoL parameters [7-9] as well as to abrupt estrogen deficiency, following chemotherapy and/or hormonal therapy, which can negatively influence a woman's sexual functioning [10].

There are many conflicting results in the literature on comparing QoL and BI following mastectomy with or without breast recon-

\section{KARGER}

Fax +497614520714

\section{(c) 2016 S. Karger GmbH, Freiburg}

$1661-3791 / 16 / 0111-0028 \$ 39.50 / 0$
Dr. Roberta Spatuzzi 
struction. Reconstructive surgery after mastectomy is generally felt to improve the women's BI perception compared with mastectomy alone [11], but other aspects of the patients' QoL and well-being do not appear to differ $[12,13]$. However, most studies that evaluated the benefit of breast reconstruction after mastectomy found no significant differences in BI between the 2 surgical procedures $[13,14]$.

An important determinant of the ability of breast cancer patients to cope with their illness and treatments is the perceived social support (PSS), which can be defined as the extent to which an individual believes that his/her needs for support, information and feedback are fulfilled [15]. The perceived availability of interpersonal resources, such as the verbal and nonverbal communication of caring and concern, including listening, 'being there', emphasizing, reassuring and comforting [16], can protect persons from potentially adverse effects of stressful events (buffering model) [17]. Therefore, for a woman who has completed treatment, the perceived social supportiveness of relationships can be considered a coping resource that could enhance the QoL and ease the transition into life after treatment [18]. Although many studies have been conducted on the role of social support for well-being, QoL, and BI in women with breast cancer $[19,20]$ there are still many aspects that need to be clarified, for example the role of PSS from different sources: family, friends, and significant others. 'Significant other' (or SO) is a generic term, also found in plural form, 'significant others', used for the first time in 1953 by H. S. Sullivan, which denotes any person who has great importance to an individual's life, well-being, and self-evaluation, such as a spouse/partner, a family member, a close friend, and/or a coworker. Our aim was to investigate and compare the effects of 3 surgical options (BCS, mastectomy alone, and mastectomy with breast reconstruction) on BI and QoL in an Italian sample of women who underwent breast surgery $[1,10]$ and, through the Multidimensional Scale of PSS (MSPSS) [21], the influence of social support on BI.

\section{Methods}

The subjects were selected from a cancer center, the Institute for Cancer Treatment and Research-Oncology Referral Center of Basilicata (IRCCS-CROB) in Rionero in Vulture (PZ), and a general hospital, the 'Madonna delle Grazie' Hospital in Matera, Italy, from January to September 2010. The eligibility was based on: Italian female patients; age 35-75 years, with previous surgical breast procedure for early-stage breast cancer (stage I-II) and with an elapsed time after surgery of less than 5 years. We excluded women with physical or cognitive disorders, previous history of psychiatric disorders, previous disfigurement (traumatic injuries, congenital defects, or prior surgical procedures), lymphedema and alopecia induced by chemotherapy, in order to exclude possible confounding factors on QoL, which could modify the perception of BI $[22,23]$.

In the clinic, 208 patients meeting the inclusion criteria were approached by the physician and asked to participate in the study. Only 198 of these patients decided to participate and signed a consensus form regarding the study protocol after detailed explanation by the physicians at the clinics. The patients were interviewed by psychologists and were administered 4 self-report questionnaires aimed at evaluating the QoL, BI, and PSS of patients (see below). During the interview, sociodemographic and clinical variables were collected. 41 patients did not answer all the questions in the questionnaires: It was therefore decided not to consider them for the analysis. The study used a sample of 157 women.

\section{Questionnaires}

EORTC Quality of Life Questionnaire (QLQ-C30)

QoL was evaluated using the Italian translation of the QLQ-C30 questionnaire (scores ranging from 0 to 100), version 3.0 of the European Organization for Research and Treatment of Cancer (EORTC) Study Group of Quality of Life [24-26], in accordance with the EORTC QLQ-C30 Scoring Manual [25]. The EORTC QLQ-C30 is composed of 5 functional subscales that assess physical functioning, role functioning, emotional functioning, cognitive functioning, and social functioning, 2 items that assess the global health status and QoL (GQL), and 9 symptom subscales/items that assess fatigue, nausea and vomiting, pain, dyspnea, insomnia, appetite loss, constipation, diarrhea, and financial difficulties. Higher average scores for a functional scale and for the GQL represent better functioning and QoL, but a high score for a symptom scale represents a high level of symptomatology.

\section{EORTC Breast Cancer Module Questionnaire (QLQ-BR23)}

The breast cancer module is designed for patients with different disease stages and treatment modalities. The module comprises 23 items, which incorporate 5 multi-item scales to assess systemic therapy side effects, arm symptoms, breast symptoms, BI, and sexual functioning. In addition, single items assess sexual enjoyment, hair loss, and future perspective [27]. Functional and symptom items of the EORTC QLQ-C30 and QLQ-BR23 questionnaires were rated on a 4-level response system from 'not at all' (score 1) to 'very much' (score 4), while the GQL (Q29, Q30) used a 7-point response scale.

\section{Body Image Scale (BIS)}

The BIS [28] consists of 10 items comprising affective items (e.g., feeling feminine, feeling attractive), behavioral items (e.g., finding it hard to look at oneself naked, avoiding people because of appearance), and cognitive items (e.g., satisfied with appearance or with scar). Respondents were asked to consider any change since diagnosis or treatment by selecting the appropriate response category for each question. All items are rated on a 4-point response scale, from 'not at all' (score 0 ) to 'very much' (score 3 ). The scores for all the 10 items were then summed up to produce an overall summary score for each patient, ranging from 0 to 30 . A score of 0 represented no symptoms/distress and higher scores represented increasing symptoms/distress. 4 items (from Q39 to Q42) from the full BIS have been incorporated into the EORTC Breast Cancer Module. The Italian version of the BIS was translated through a forward-backward procedure of translation by Didier et al. [29], according to the recommendations for translating questionnaires of the QoL Group of the EORTC [30], and is actually in a validation study.

\section{Multidimensional Scale of Perceived Social Support (MSPSS)}

The MSPSS [21] is a 12-item scale with 4 items for each subscale evaluating 3 sources of support: the family subscale (e.g., 'I get the emotional help and support I need from my family'), the friends subscale (e.g., 'I can talk about my problems with my friends'), and the significant other subscale (e.g., 'I have a special person who is a real source of comfort to me'). Each item is rated on a 7 -point scale ranging from 'disagree very strongly' (1) to 'agree very strongly' (7). The scores on the 4 items under each subscale were combined to obtain the subscale score (range 4-28) and all subscale scores were added to obtain the total scale score (range 12-84). Higher scores indicated higher PSS. An Italian version of the MSPSS was used for this study [31].

\section{Statistics}

The patients were stratified into 3 different treatment groups according to the surgical procedure they underwent: 'BCS', 'Mastectomy' (M), and 'Reconstruction after mastectomy' (R).

We chose to treat the immediate- and delayed-reconstruction patients as one single group after the independent-sample t-test analysis, because we found no differences in all scales considered. Patients were stratified according to age, time since surgery (we used it continuously), marital status (single vs. not single), educational status (low scholar grade vs. high scholar grade), menopausal 
Table 1. Sociodemographic and clinical characteristics and PSS results (evaluated through the MSPSS) in the total patient population and in the different surgical groups

\begin{tabular}{|c|c|c|c|c|c|}
\hline Variables & $\begin{array}{l}\text { All patients } \\
(\mathrm{n}=157)\end{array}$ & $\begin{array}{l}\text { BCS group } \\
(\mathrm{n}=72,46 \%)\end{array}$ & $\begin{array}{l}\text { M group } \\
(\mathrm{n}=44,28 \%)\end{array}$ & $\begin{array}{l}\text { R group } \\
(\mathrm{n}=41,26 \%)\end{array}$ & $\mathrm{p}$ \\
\hline Age in years, mean $(\mathrm{SD})$ & $56.4(12.7)$ & $57.6(12.0)$ & $61.3(14.0)$ & $49.3(9.2)$ & $<0.001$ \\
\hline Time since surgery in years, median (range) & $1.0(0-5)$ & $0.8(0-4)$ & $1.0(0-5)$ & $0.8(0-5)$ & 0.184 \\
\hline Education, $\mathrm{N}(\%)$ & & & & & 0.008 \\
\hline Low scholar grade & $99(63.1)$ & $48(66.7)$ & $33(75.0)$ & $18(43.9)$ & \\
\hline High scholar grade & $58(36.9)$ & $24(33.3)$ & $11(25.0)$ & $23(56.1)$ & \\
\hline Marital status, $\mathrm{N}(\%)$ & & & & & 0.827 \\
\hline Single & $38(24.2)$ & $16(22.2)$ & $12(27.3)$ & $10(24.4)$ & \\
\hline Not single & $119(75.8)$ & $56(77.8)$ & $32(72.7)$ & $31(75.6)$ & \\
\hline Menopausal status, N (\%) & & & & & 0.133 \\
\hline Premenopausal & $30(19.1)$ & $10(13.9)$ & $8(18.2)$ & $12(29.3)$ & \\
\hline Postmenopausal & $127(80.9)$ & $62(86.1)$ & $36(81.8)$ & $29(70.7)$ & \\
\hline \multicolumn{6}{|l|}{ Adjuvant treatment post-surgery, $\mathrm{N}(\%)$} \\
\hline Chemotherapy & & & & & 0.019 \\
\hline Yes & $103(65.6)$ & $41(56.9)$ & $28(63.6)$ & $34(82.9)$ & \\
\hline No & $54(34.4)$ & $31(43.1)$ & $16(36.4)$ & $7(17.1)$ & \\
\hline Radiation therapy & & & & & $<0.001$ \\
\hline Yes & $105(66.9)$ & $64(88.9)$ & $26(59.1)$ & $15(36.6)$ & \\
\hline No & $52(33.1)$ & $8(11.1)$ & $18(40.9)$ & $26(63.4)$ & \\
\hline Hormone therapy & & & & & 0.046 \\
\hline Yes & $97(61.8)$ & $52(72.2)$ & $23(52.3)$ & $22(53.7)$ & \\
\hline No & $60(38.2)$ & $20(27.8)$ & $21(47.7)$ & $19(46.3)$ & \\
\hline \multicolumn{6}{|l|}{ PSS, mean (SD) } \\
\hline Family & $23.8(5.8)$ & $25.1(4.7)$ & $21.9(7.1)$ & $23.5(5.4)$ & 0.016 \\
\hline Friends & $19.7(7.1)$ & $21.7(6.3)$ & $16.5(7.3)$ & $19.8(7.3)$ & 0.001 \\
\hline Significant other & $22.9(6.5)$ & $24.6(4.8)$ & $20.4(8.2)$ & $22.5(6.2)$ & 0.003 \\
\hline Total scale score & $66.5(16.4)$ & $71.5(12.4)$ & $58.9(19.8)$ & $65.9(15.6)$ & $<0.001$ \\
\hline
\end{tabular}

ANOVA (analysis of variance): F-test for continuous variables, chi-square test for categorical variables. PSS $=$ Perceived social support, MSPSS $=$ Multidimensional Scale of Perceived Social Support, BCS $=$ breast-conserving surgery, $\mathrm{M}=$ mastectomy, $\mathrm{R}=$ reconstruction after mastectomy, $\mathrm{SD}=$ standard deviation status and type of adjuvant treatment post-surgery. Statistical evaluations were made using the chi-square $\left(\chi^{2}\right)$ test for categorical variables, the one-way analysis of variance (ANOVA) with pairwise post-hoc comparisons (Scheffé), the independent samples t-test, and Pearson's coefficient. Multivariate linear regression was fitted to adjust for covariates in order to assess the independent effects of the surgical procedures on QoL and BI in the presence of possible confounders. Data were analyzed using the SPSS ${ }^{\circledR}$ statistical software package (SPSS, Chicago, IL, USA) version 17.0 for Windows ${ }^{\circledR}$. All tests were considered significant at $\mathrm{p}$-values $\leq 0.05$.

\section{Results}

\section{Sociodemographic and Clinical Characteristics}

The sociodemographic and relevant clinical characteristics of the surgical groups are shown in table 1. Patients in the BCS group underwent lumpectomy (14\%) and conservative surgery (86\%). The M group included patients treated with modified radical mastectomy (67\%), radical mastectomy (26\%), total mastectomy (4\%), and nipple-areola complex (NAC)-sparing mastectomy (3\%). In the R group, 23 (56\%) patients had immediate breast reconstruction whereas 18 (44\%) patients had delayed breast reconstruction. The patients decided to undergo breast reconstruction after having been fully informed about the possible outcomes of surgical treat- ment. The most frequently reported reasons given by the reconstruction group, for having reconstruction, included: to get rid of the external breast prosthesis, to regain femininity. The least influential factors were to improve marital and sexual relations.

The 3 groups were different in age $\left(\mathrm{F}_{(2.154)}=11.011, \mathrm{p}<0.001\right)$, with patients in the $\mathrm{R}$ group being younger than those in the $\mathrm{M}(\mathrm{p}$ $<0.001)$ and BCS ( $\mathrm{p}=0.003)$ groups. We also found a higher education level in the R group compared with the $\mathrm{M}$ and BCS groups $\left(\chi_{(2)}^{2}=9.55, p=0.008\right)$. Women in the $\mathrm{R}$ group underwent chemotherapy more often than patients in the other groups $\left(\chi_{(2)}^{2}=7.921\right.$, $\mathrm{p}=0.019$ ), women in the BCS and M groups underwent radiation therapy more frequently than patients in the $\mathrm{R}$ group $\left(\chi_{(2)}^{2}=\right.$ $33.937, \mathrm{p}<0.001)$, and women in the BCS group underwent hormone treatment more often than women in the $\mathrm{M}$ and $\mathrm{R}$ groups $\left(\chi_{(2)}^{2}=6.155, p=0.046\right)$. No differences were observed in terms of marital and menopausal status.

\section{Quality of Life}

Supplemental Table 1 (online supplemental, www.karger. $\mathrm{com} /$ ?DOI=443493) shows the values of the functional and symptom scales of the EORTC QLQ-C30 and QLQ-BR23 questionnaires. The mean values of the GQL scale and of the functional 
scales of the QLQ-C30 were all $\geq 60$. The symptom scales of the QLQ-C30 in general showed a low degree of symptomatology. The most frequent complaints for all 3 surgical groups were insomnia, fatigue, pain, and financial difficulties, whereas nausea and vomiting, diarrhea, appetite loss, and constipation were rarely reported. There was no difference among the groups with respect to GQL, most of the functioning scales, and all the items in the symptom scale. We found differences among the 3 groups in physical functioning $\left(\mathrm{F}_{(2.154)}=3.068, \mathrm{p}=0.049\right)$ but no differences in terms of post-hoc pairwise comparisons. Therefore, we considered differences among the groups in the role functioning $\left(\mathrm{F}_{(2.154)}=3.500, \mathrm{p}\right.$ $=0.033)$ and dyspnea $\left(\mathrm{F}_{(2.154)}=3.727, \mathrm{p}=0.026\right)$ scales. Post-hoc pairwise comparisons showed that patients in the BCS group had fewer difficulties with role functioning than those in the M group $(\mathrm{p}=0.034)$. This result remained significant even after adjustment for age, education, and type of adjuvant treatment post-surgery. After adjusting for the above covariates, the patients in the R group did not show a different degree of dyspnea than those who had mastectomy alone. The results regarding the QLQ-BR23 items showed no differences among all symptom scales/items and for most of the functioning items, but differences existed in the BI scale $\left(\mathrm{F}_{(2.154)}=4.571, \mathrm{p}=0.012\right)$ : Patients in the BCS group had a better $B I$ when compared with the $M(p=0.039)$ and $R(p=0.055)$ groups. There was no difference between the $\mathrm{M}$ and $\mathrm{R}$ groups ( $\mathrm{p}>$ $0.05)$. All the results were unaffected after controlling for the same adjustment as above.

\section{Body Image}

After adjusting for age, education, and type of adjuvant treatment post-surgery, the differences among the 3 surgical treatment groups are significant. The results are as follows: Regarding the BI appraisal with the BIS, the differences observed among the 3 surgical procedures $\left(\mathrm{F}_{(2.154)}=7.94, \mathrm{p}<0.001\right)$ confirmed the BI scale results obtained with the EORTC QLQ-BR23 (Supplemental Table 1; online supplemental, www.karger.com/?DOI=443493). We found that women having BCS reported fewer difficulties with their BI compared with the $M(p=0.002)$ and $R(p=0.021)$ groups. There were no differences in the BIS scores between the $\mathrm{M}$ and $\mathrm{R}$ groups ( $p>0.05$ ), even after adjusting for covariates.

\section{Perceived Social Support}

In the PSS evaluation with the MSPSS total scale score (Table 1), we found differences among the 3 surgical groups $\left(\mathrm{F}_{(2.154)}=8.810\right.$, $\mathrm{p}=0.001)$. Post-hoc pairwise comparisons showed that the women in the BCS group perceived more social support than the women in the $M$ group $(p<0.001)$. Differences existed between the surgical procedures regarding all MSPSS subscale scores (MSPSS-family/ friends and -significant other) in favor of BCS: MSPSS-family $\left(\mathrm{F}_{(2.154)}=4.243, \mathrm{p}=0.016\right)$, MSPSS-friends $\left(\mathrm{F}_{(2.154)}=7.611, \mathrm{p}<\right.$ $0.001)$, and MSPSS-significant other $\left(\mathrm{F}_{(2.154)}=6.217, \mathrm{p}=0.003\right)$. We found a negative correlation between a positive PSS and major alterations in the BI $(\mathrm{r}=-0.343, \mathrm{p}<0.05)$ in the R group. Considering the MSPSS subscale scores, we observed, in this group, a negative correlation between the women's BIS scores and their
MSPSS-family scores $(r=-0.401, p<0.001)$ and MSPSS-significant other scores $(\mathrm{r}=-0.485, \mathrm{p}<0.01)$, but no correlation was found between the women's BIS scores and their MSPSS-friends subscale scores. The M and BCS groups did not feel the effects of PSS on BI perception. The results obtained for women with breast reconstruction were still significant after controlling for age, time since surgery, education level, and type of adjuvant treatment postsurgery.

In the analysis of the BIS scores, adjusted for PSS from family, friends and significant other, the differences between the BCS and the non-BCS groups remained significant $(\mathrm{p}<0.05)$. The comparison between the $\mathrm{M}$ and the $\mathrm{R}$ group for $\mathrm{BI}$ remained not significant after controlling for the same variables as above.

\section{Discussion}

Our findings support previous research suggesting that many QoL variables do not differ by surgery methods $[3,6,7,12,13]$. In line with Engel et al. [32], we found that women with mastectomy had more limited role functioning than those in the BCS group. In the symptom domain, evaluated with the EORTC QLQ-C30 and QLQ-BR23, we found differences among the surgical groups only for the scale of dyspnea. We also found that, in our sample of Italian women, BCS demonstrated superiority in BI perception when compared to non-conservative surgery. This result is supported by the international literature $[11,14]$. In addition, women with breast reconstruction did not report different difficulties with their BI than women who had mastectomy alone $[12,33]$. PSS might play a fundamental role in determining the type of reaction to surgery.

The results of this study provide an understanding of the importance of social support, especially from family and significant others, for women who underwent breast surgery. The findings show that, for women who decided to have reconstruction after mastectomy, social support from family and significant others could be involved in the level of satisfaction with their BI following breast surgery. Women who decided to have breast reconstruction after mastectomy feel more the buffering role of PSS regarding their BI. As reported by Helgeson and Cohen [16], interventions that involve family and partners should be aimed at improving communication and facilitating both the patients' and the family members' expressions of needs and feelings. Our study had some limitations. Specifically, the sample is relatively small and we did not consider the women's baseline premorbid BI, which may all have strongly influenced the QoL and BI. Little is known about the extent to which women in the 3 treatment groups had input into the choice of their surgery treatment and on what basis they might have made these decisions. This leaves open the possibility that differences in outcome variables may be driven by selection factors rather than the type of surgical treatments received. Despite the limitations, our findings indicate the importance that healthcare professionals ascribe to the role of emotional support from the partner and family members in the women's experiences of illness and surgery treatments, from their own experiences during this pe- 
riod. BI is a complex, dynamic, and multidimensional construct that keeps evolving over a woman's life. As such, it cannot be separated from considerations concerning the patient's overall personality, social and cultural background, as well as the network of her personal relationships.

\section{Conclusions}

In conclusion, a more comprehensive approach to women with breast cancer who choose to have reconstruction after mastectomy should be implemented, with a preliminary psychosocial evaluation of the patients' characteristics and their main sources of support, particularly from their family and significant others (e.g., spouse/partner). This type of evaluation could be predictive of the satisfaction level of a woman's BI after breast surgery and could help healthcare professionals in programming interventions to provide psychological support for women and their families and/or partners before and after surgical treatment.

\section{Supplemental Table}

Supplemental Table 1. Differences in quality of life and body image evaluated by QLQ-C30, QLQ-BR23, and BIS across treatment groups

To access the online supplemental table, please refer to $w w w$.karger. com/?DOI=443493.

\section{Disclosure Statement}

The authors have no conflict of interest to declare.

\section{References}

1 Caffo O, Amichetti M, Ferro A, et al.: Pain and quality of life after surgery for breast cancer. Breast Cancer Res Treat 2003;80:39-48.

2 Harding MM: Incidence of distress and associated factors in women undergoing breast diagnostic evaluation. West J Nurs Res 2014;36:475-494.

3 Nissen MJ, Swenson KK, Ritz LJ, et al.: Quality of life after breast carcinoma surgery: a comparison of three surgical procedures. Cancer 2001;91:1238-1246.

$\checkmark 4$ Rhondali W, Chisholm GB, Filbet M, et al.: Screening for body image dissatisfaction in patients with advanced cancer: a pilot study. J Palliat Med 2015;18: 151-156.

5 Fingeret MC, Nipomnick S, Guindani M, et al.: Body image screening for cancer patients undergoing reconstructive surgery. Psychooncology 2014;23:898-905.

6 Reaby LL, Hort LK: Postmastectomy attitudes in women who wear external breast prostheses compared to those who have undergone breast reconstructions. Behav Med 1995;18:55-67.

7 Kenny P, King MT, Shiell A, et al.: Early stage breast cancer: costs and quality of life one year after treatment by mastectomy or conservative surgery and radiation therapy. Breast 2000;9:37-44.

8 Amichetti M, Caffo O: Quality of life in patients with early stage breast carcinoma treated with conservation surgery and radiotherapy. An Italian monoinstitutional study. Tumori 2001;87:78-84.

9 Hopwood P, Haviland J, Mills J, et al.: The impact of age and clinical factors on quality of life in early breast cancer: an analysis of 2208 women recruited to the UK START Trial (Standardization of Breast Radiotherapy Trial). Breast 2007; 16:241-251.

10 Biglia N, Moggio G, Peano E, et al.: Effects of surgical and adjuvant therapies for breast cancer on sexuality, cognitive functions and body weight. J Sex Med 2010 7:1891-1900.

11 Al-Ghazal SK, Fallowfield L, Blamey RW: Comparison of psychological aspects and patient satisfaction following breast conserving surgery, simple mastectomy and breast reconstruction. Eur J Cancer 2000;36:19381943.

12 Avis NE, Crawford S, Manuel J: Psychosocial problems among younger women with breast cancer. Psychooncology 2004;13:295-308.
3 Rowland JH, Desmond KA, Meyerowitz BE, et al.: Role of breast reconstructive surgery in physical and emotional outcomes among breast cancer survivors. J Natl Cancer Inst 2000;92:1422-1429.

14 Yurek D, Farrar W, Andersen BL: Breast cancer surgery: comparing surgical groups and determining individual differences in postoperative sexuality and body change stress. J Consult Clin Psychol 2000;68:697-709.

15 Chang O, Choi EK, Kim IR, et al.: Association between socioeconomic status and altered appearance distress, body image, and quality of life among breast cancer patients. Asian Pac J Cancer Prev 2014;15:8607-8612.

16 Helgeson VS, Cohen S: Social support and adjustment to cancer: reconciling descriptive, correlational, and intervention research. Health Psychol 1996;15:135148 .

17 Cohen S, Wills TA: Stress, social support, and the buffering hypothesis. Psychol Bull 1985;98:310-357.

18 Leedham B, Ganz PA: Psychosocial concerns and quality of life in breast cancer survivors. Cancer Invest 1999; 17:342-348.

19 Champion VL, Wagner LI, Monahan PO, et al.: Comparison of younger and older breast cancer survivors and age-matched controls on specific and overall quality of life domains. Cancer 2014;120:2237-2246.

20 Michael YL, Berkman LF, Colditz GA, et al.: Social networks and health-related quality of life in breast cancer survivors: a prospective study. J Psychosom Res 2002; 52:285-293.

21 Zimet GD, Dahlem NW, Zimet SG, Farley GK: The Multidimensional Scale of Perceived Social Support. J Pers Assess 1988:52:30-41.

22 McGarvey EL, Baum LD, Pinkerton RC, Rogers LM: Psychological sequelae and alopecia among women with cancer. Cancer Pract 2001;9:283-289.

23 Velanovich V, Szymanski W: Quality of life of breast cancer patients with lymphedema. Am J Surg 1999; 177:184-187.

24 Aaronson NK, Ahmedzai S, Bergman B, et al.: The European Organization for Research and Treatment of Cancer QLQ-C30: a quality of life instrument for use in international clinical trials in oncology. J Natl Cancer Inst 1993;85:365-375.
25 Fayers PM, Aaronson NK, Bjordal K, Groenvold M Curran D, Bottomley A; on behalf of the EORTC Quality of Life Study Group: The EORTC QLQ-C30 Scoring Manual, ed 3. Brussels, EORTC Quality of Life Group Publications, 2001.

26 McLachlan SA, Devins GM, Goodwin PJ: Validation of the European Organization for Research and Treatment of Cancer Quality of Life Questionnaire (QLQC30) as a measure of psychosocial function in breast cancer patients. Eur J Cancer 1998;34:510-517.

27 Sprangers MA, Groenvold M, Arraras JI, Franklin J, te Velde A, et al.: The European Organization for Research and Treatment of Cancer breast cancer-specific quality-of-life questionnaire module: first results from a three-country field study. J Clin Oncol 1996;14: 2756-2768.

28 Hopwood P, Fletcher I, Lee A, Al-Ghazal S: A body image scale for use with cancer patients. Eur J Cancer 2001;37:189-197.

29 Didier F, Radice D, Gandini S, et al.: Does nipple preservation in mastectomy improve satisfaction with cosmetic results, psychological adjustment, body image and sexuality? Breast Cancer Res Treat 2009;118:623633 .

30 Cull A, Sprangers N, Bjordal K, Aaronson NK, West K, Bottomley A; on behalf of the EORTC Quality of Life Study Group: EORTC Quality of Life Group Translation Procedure, ed 2. Brussels, EORTC Quality of Life Group Publications, 2002.

31 Prezza M, Pacilli MG: Perceived social support from significant others, family and friends and several sociodemographic characteristics. J Commun Appl Soc Psychol 2002; 12:422-429.

32 Engel J, Kerr J, Schlesinger-Raab A, Sauer H, Holzel D: Quality of life following breast-conserving therapy or mastectomy. Results of a 5-year prospective study. Breast 2004;10:223-231.

33 Lee C, Sunu C, Pignone M: Patient-reported outcomes of breast reconstruction after mastectomy: a systematic review. J Am Coll Surg 2009;209:123-133. 